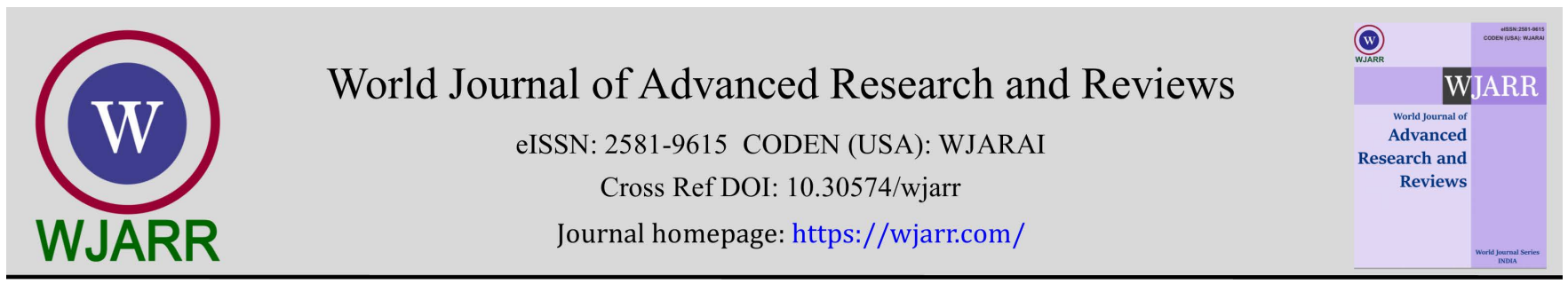

(RESEARCH ARTICLE)

Check for updates

\title{
Gingival zenith evaluation in an Andean population from Ecuador
}

Diego Mauricio Bravo-Calderón 1, ${ }^{*}$, María Fernanda Vera-Procel ${ }^{1}$, Daniela Andrea Molina-Koppel 1, Pamela Estefania Bernal-Paucar ${ }^{1}$, María José Vásquez-Vélez ${ }^{1}$, Mariana Mora Oleas ${ }^{2}$ and Manuel Estuardo BravoCalderón 1,2

${ }^{1}$ School of Dentistry, University of Cuenca, Cuenca, Azuay, Ecuador.

2 Private Dental Clinic, Cuenca, Azuay, Ecuador.

World Journal of Advanced Research and Reviews, 2021, 09(02), 095-102

Publication history: Received on 21 January 2021; revised on 11 February 2021; accepted on 13 February 2021

Article DOI: https://doi.org/10.30574/wjarr.2021.9.2.0046

\begin{abstract}
Objectives: The aim of this study was to evaluate the characteristics of the gingival zenith of the maxillary incisors, canines and premolars in an Andean population from Ecuador.

Materials and methods: A total of 51 subjects ( 30 men and 21 women; mean age $=21$ years) with healthy gingival tissue were recruited. Digital study models were obtained from maxillary casts and the features of the gingival zeniths including its position, gingival lines and level in the lateral incisors were analyzed.

Results: The gingival zenith was distally displaced in all the maxillary central and lateral incisors and in 70.59\%; 62.74\% and $91.17 \%$ of the canines, first and second premolars, respectively. For the maxillary anterior teeth, the greater mean of displacement was verified in central incisors $(0.89 \mathrm{~mm} \pm 0.27 \mathrm{SD})$, followed by the lateral incisors $(0.58 \mathrm{~mm} \pm 0.22 \mathrm{SD})$ and the canines $(0.48 \mathrm{~mm} \pm 0.21 \mathrm{SD})$. The displacement levels in the central incisors were significantly higher in females compared to male participants $(p=0.037)$. In addition, the ascending gingival line was the most common in the anterior dentition (right: 52.9\%; left: 51\%), whereas the descending one was the most prevalent in the premolar area (right: 58.8\%; left: 70.6\%). Lastly, the gingival zeniths of lateral incisors were more frequently located coronal to the gingival line $(86.27 \%)$, to a mean distance of $0.82 \mathrm{~mm}(0.1-3.1 \mathrm{~mm} ; \pm 0.46 \mathrm{SD})$ and without significant differences among the genders $(p=0.520)$.
\end{abstract}

Conclusion: The data obtained from the present study could be taken into consideration during dental esthetic treatments, especially for Andean patients.

Keywords: Gingiva; Gingival Zenith; Maxillary Teeth; Dental Esthetics

\section{Introduction}

An esthetically pleasing smile is determined by harmony between the teeth, the lips and the gingiva[1-3]. Regarding the optimal gingival architecture, it has been conventionally described as having a scalloped contour around the surfaces of the tooth, following the course of the cement-enamel junction. Therefore, is concave apically in the free surfaces and convex occlusally at the tip of the papilla [4-6]. The gingival zenith (GZ) is defined as the most apical point of the gingival marginal scallop and can be located either mesial, distal, or in coincidence with the long axis of each tooth[3,5,7-9].

\footnotetext{
${ }^{*}$ Corresponding author: Diego Mauricio Bravo-Calderón

School of Dentistry, University of Cuenca, Cuenca, Azuay, Ecuador.

Copyright $(2021$ Author(s) retain the copyright of this article. This article is published under the terms of the Creative Commons Attribution Liscense 4.0.
} 
Meanwhile, the gingival line (GL), also named as gingival esthetic line, represents the tangent connecting the gingival zeniths of the central incisor and the canine tooth and is classified as straight when the GZ points of the central incisor and the canine tooth are parallel, ascending when the GZ of the canine is located apically to the central incisor and descending when the GZ of the canine is coronal to the central incisor [10]. Finally, the gingival zenith level (GZL) is the distance between the lateral incisor zenith and the GL, being that this zenith can be located coronal, apical or in coincidence with the GL $[2,7]$.

Considering their great impact on the esthetics of the smile, these anatomical points have been studied in different populations. However, analyzes in Andean populations from Ecuador have not been performed. In addition, there are little scientific information about their location in premolars[11]. Therefore, the aim of this study was to analyze the GZ and the esthetic variables that revolve around it such as: location, line and level in the maxillary incisors, canines and premolars of a sample from Cuenca, Azuay, Ecuador.

\section{Material and methods}

\subsection{Study population}

The universe of this cross-sectional study was constituted by 377 students of the School of Dentistry, University of Cuenca, Cuenca, Ecuador and the final sample was of 51 individuals (30 males and 21 females), 17 to 26 years of age (mean 21 years \pm 0.497 standard deviation (SD)) who had permanent incisors, canines and premolars. Students under orthodontic treatment with periodontal alterations (probing deep $>3 \mathrm{~mm}$, attachment loss, gingival hyperplasia, gingival recession or altered passive eruption), having crowding or restorative treatment in the interest teeth or with past history of orthodontics or periodontal surgical procedures (gingivoplasty, gingivectomy or crown enlargement), were excluded of the study.

The Research Committee of the School of Dentistry, University of Cuenca, Cuenca, Ecuador approved this study (UCDIFO-PROY-17-009) and the written informed consent was obtained from all participants.

\subsection{Gingival zenith analyses}

Dental impressions using alginate (Tulip, Cavex Holland BV, Haarlem, Netherlands) mixed with water (ratio 1:1) were taken from each participant. Then the study casts were created by pouring in the alginate impressions with Type IV dental stone (Whip Mix Corporation, Louisville, KY, EE. UU.) mixed with water in a 2:1 ratio.

In sequence, the study casts were labeled and digitalized with the CEREC Bluecam scanner (Sirona Dental Systems, Bensheim, Germany). The digitized models were analyzed using ImageJ 1.46r software (National Institutes of Health, Bethesda, Maryland, USA) (Figure 1). Two points of reference were established: one being the line along the teeth longitudinal axis and the GZ, that was considered as the highest apical point of the gingival margin. Each GZ was evaluated according to its position (in coincidence with the respective tooth long axis or displaced towards the mesial or distal) and to the amount of displacement in millimeters.

Anterior and posterior gingival lines were determined connecting the GZ of the central incisor with the GZ of the ipsilateral canine and drawing a line between the GZ of the premolars, respectively. In addition, both lines was classified as straight, ascending, or descending, being that for the anterior GL were employed the aforementioned parameters [10]. Meanwhile, the posterior GL was considered as straight when the zeniths of the premolars were parallels, ascending when the GZ of the second premolar was apically located and descending when the GZ of the second premolar was coronal to the first premolar.

Finally, the coincidence or the coronal/apical displacement in millimeters of the GZ of the lateral incisors relative to the adjacent anterior gingival line were verified in order to establish the GZLs of these teeth.

All variables were evaluated for each maxillary quadrant and the measurements were performed by a single investigator without knowledge of the gender of the participants. 


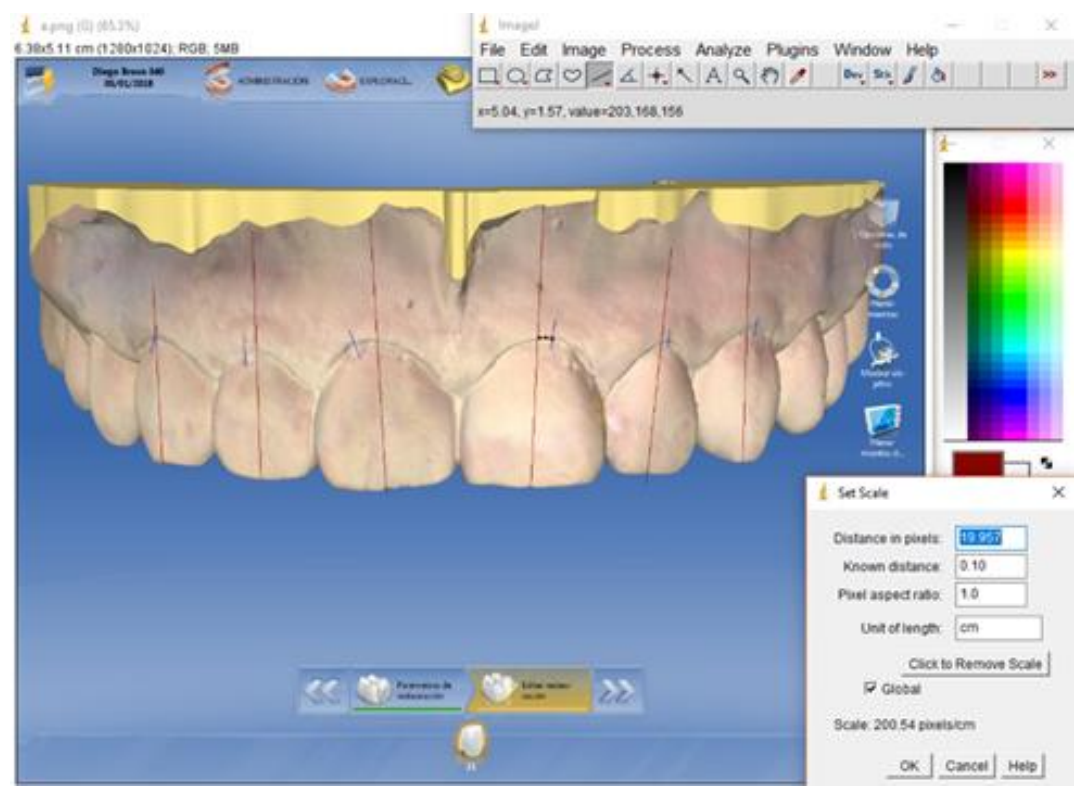

Figure 1 Gingival zenith evaluation in digital study models through Image $1.46 \mathrm{r}$ software.

\subsection{Statistical analyses}

Statistical analyzes were obtained through the IBM SPSS Statistics version 20.0 (IBM Corporation, Armonk, NY, EEUU). The zenith characteristics were submitted to descriptive statistical analysis. Additionally, the distances between the zeniths and tooth long axis or to anterior gingival line (for lateral incisors) were analyzed through the KolmogorovSmirnov normal distribution test and then comparisons of these variables based on the gender of the students were performed using the Mann-Whitney test. Levels equal or inferior to $5 \%(p \leq 0,05)$ were considered to represent a statistically significant result.

\section{Results}

\subsection{Location and distance of the gingival zenith to the tooth long axis}

The results of the location of the GZ in relation to the tooth long axis and of the amount of displacement are summarized in Table 1 and 2, respectively.

Table 1 Gingival zenith location in relation to the long axis of maxillary incisors, canines and premolars

\begin{tabular}{|c|c|c|c|c|c|c|c|}
\hline \multicolumn{2}{|l|}{ Tooth } & \multicolumn{6}{|c|}{ Gingival Zenith Location } \\
\hline & & \multicolumn{2}{|c|}{ Coincidence } & \multicolumn{2}{|c|}{ Mesial } & \multicolumn{2}{|c|}{ Distal } \\
\hline & & $\mathbf{N}$ & $\%$ & $\mathbf{N}$ & $\%$ & $\mathbf{N}$ & $\%$ \\
\hline \multirow[t]{2}{*}{ Central Incisor } & 1.1 & 0 & 0 & 0 & 0 & 51 & 100 \\
\hline & 2.1 & 0 & 0 & 0 & 0 & 51 & 100 \\
\hline \multirow[t]{2}{*}{ Lateral Incisor } & 1.2 & 0 & 0 & 0 & 0 & 51 & 100 \\
\hline & 2.2 & 0 & 0 & 0 & 0 & 51 & 100 \\
\hline \multirow[t]{2}{*}{ Canine } & 1.3 & 14 & 27.5 & 0 & 0 & 37 & 72.5 \\
\hline & 2.3 & 16 & 31.4 & 0 & 0 & 35 & 68.6 \\
\hline \multirow[t]{2}{*}{ First Premolar } & 1.4 & 0 & 0 & 17 & 33.3 & 34 & 66.7 \\
\hline & 2.4 & 0 & 0 & 21 & 41.2 & 30 & 58.7 \\
\hline \multirow[t]{2}{*}{ Second Premolar } & 1.5 & 0 & 0 & 1 & 2 & 50 & 98 \\
\hline & 2.5 & 0 & 0 & 8 & 15.7 & 43 & 84.3 \\
\hline
\end{tabular}

The GZ on all the central and lateral incisors exhibited a distal displacement that ranged from 0.44 to $1.8 \mathrm{~mm}$ (mean $0.89 \mathrm{~mm} \pm 0.27 \mathrm{SD}$ ) and from 0.2 to 1.77 (mean $0.58 \mathrm{~mm} \pm 0.22 \mathrm{SD}$ ), respectively. Similarly, $70.59 \%$ of canines showed 
that the zenith was distally displaced in a range from 0.1 to $1 \mathrm{~mm}$ (mean $0.48 \mathrm{~mm} \pm 0.21 \mathrm{SD}$ ). Meanwhile, in 30 canines the GZ were in coincidence with the tooth long axis and none showed mesial displacement.

Specifically, in the premolars it was found that the zeniths of 64 first premolars and 93 second premolars were also distally located with values from 0.2 to $0.3 \mathrm{~mm}$ (mean $0.56 \mathrm{~mm} \pm 0.22 \mathrm{SD}$ ) and between 0.2 and $1.2 \mathrm{~mm}$ (mean $0.57 \mathrm{~mm}$ $\pm 0.21 \mathrm{SD}$ ), respectively. Only the zeniths of 38 first premolars and 9 second premolars showed mesial displacement and none had concordance with the crown long axis.

Table 2 Displacement of the gingival zenith with respect to the tooth long axis of maxillary incisors, canines and premolars.

\begin{tabular}{|c|c|c|c|c|c|c|}
\hline \multirow{3}{*}{\multicolumn{2}{|c|}{ Tooth }} & \multicolumn{5}{|c|}{ Gingival Zenith Displacement } \\
\hline & & \multicolumn{3}{|l|}{ Tooth } & \multicolumn{2}{|l|}{ Dental Group } \\
\hline & & $\begin{array}{l}\text { Minimum } \\
\text { Maximum (mm) }\end{array}$ & Mean $(\mathrm{mm})$ & SD & Mean (mm) & SD \\
\hline \multirow[t]{2}{*}{ Central Incisor } & 1.1 & $0.4-1.8$ & 0.88 & 0.28 & \multirow[t]{2}{*}{0.89} & \multirow[t]{2}{*}{0.27} \\
\hline & 2.1 & $0.5-1.7$ & 0.90 & 0.26 & & \\
\hline \multirow[t]{2}{*}{ Lateral Incisor } & 1.2 & $0.2-1$ & 0.55 & 0.19 & \multirow[t]{2}{*}{0.58} & \multirow[t]{2}{*}{0.22} \\
\hline & 2.2 & $0.2-1.3$ & 0.62 & 0.25 & & \\
\hline \multirow[t]{2}{*}{ Canine } & 1.3 & $0.1-0.8$ & 0.42 & 0.21 & \multirow[t]{2}{*}{0.48} & \multirow[t]{2}{*}{0.21} \\
\hline & 2.3 & $0.2-1$ & 0.54 & 0.21 & & \\
\hline \multirow[t]{2}{*}{ First Premolar } & 1.4 & $0.2-1.3$ & 0.54 & 0.23 & \multirow[t]{2}{*}{0.56} & \multirow[t]{2}{*}{0.22} \\
\hline & 2.4 & $0.2-1.1$ & 0.58 & 0.22 & & \\
\hline \multirow[t]{2}{*}{ Second Premolar } & 1.5 & $0.2-1.2$ & 0.58 & 0.25 & \multirow[t]{2}{*}{0.57} & \multirow[t]{2}{*}{0.21} \\
\hline & 2.5 & $0.2-1.2$ & 0.57 & 0.18 & & \\
\hline
\end{tabular}

The comparisons of the GZ position between male and female indicated statically significant differences only in central incisors ( $p=0.037$ ) in which the mean of displacement was greater in female (mean $0.95 \mathrm{~mm} \pm 0.30 \mathrm{SD}$ ) than in male participants (mean $0.82 \mathrm{~mm} \pm 0.19 \mathrm{SD}$ ) (Figure 2).

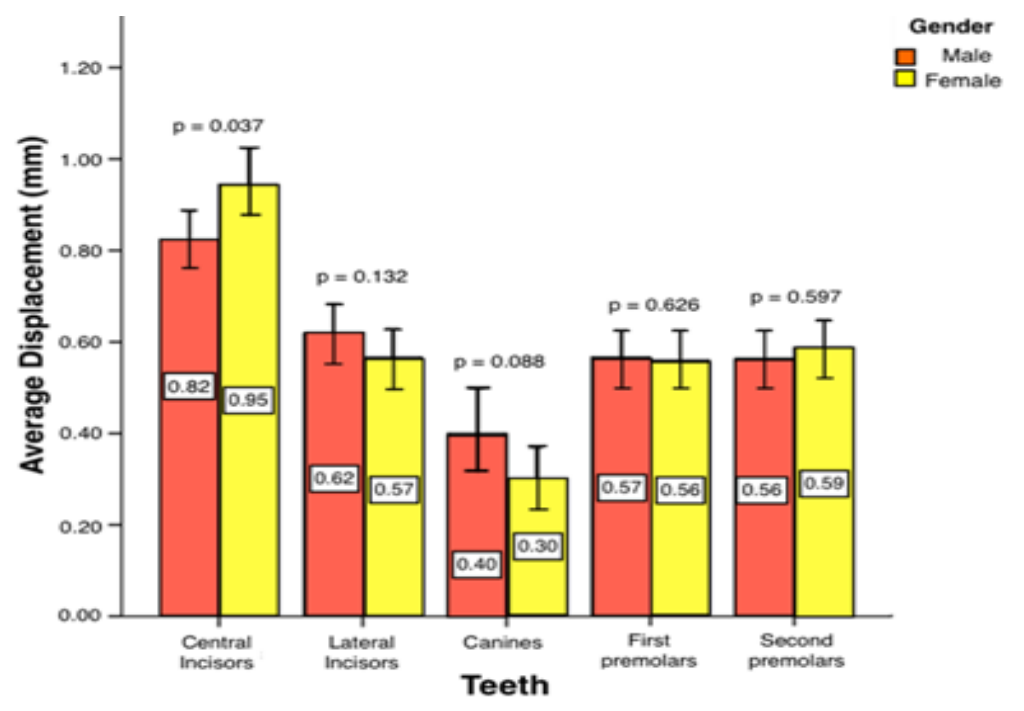

Figure 2 Gender based comparisons of the gingival zenith displacement. 


\subsection{Characterization of the gingival line}

As shown in Table 3, the characterization of the GL for each maxillary quadrant in the anterior region revealed that the ascending line was the most common (right: 52.9\%; left: 51\%), followed by the straight line (right: $31.4 \%$; left: $23.5 \%$ ) and the descending one (right: 15.7\%; left: 25.5). On the other hand, when analyzed the posterior GL, which connects the zeniths of the premolars, it was verified that the most common pattern was the descending (right: 58.8\%; left: 70.6\%), followed by the straight (right: $37.7 \%$; left: $23.5 \%$ ) and the ascending (right: 3.9\%; left: 5.9\%) (Table 3).

Table 3 Frequency of anterior and posterior gingival lines

\begin{tabular}{|l|l|l|l|l|l|l|l|l|}
\hline \multirow{2}{*}{ Gingival Line } & \multicolumn{4}{l}{ Anterior } & \multicolumn{2}{l|}{ Posterior } \\
\cline { 2 - 10 } & \multicolumn{2}{|l|}{ Right } & Left & Right & Left \\
\cline { 2 - 10 } & N & \% & N & \% & N & \% & N & $\%$ \\
\hline Straight & 16 & 31.4 & 12 & 23.5 & 19 & 37.3 & 12 & 23.5 \\
\hline Ascending & 27 & 52.9 & 26 & 51.0 & 2 & 3.9 & 3 & 5.9 \\
\hline Descending & 8 & 15.7 & 13 & 25.5 & 30 & 58.8 & 36 & 70.6 \\
\hline
\end{tabular}

\subsection{Characterization of the gingival zenith level}

Figure 3 shows the different relationship between the GZs of the lateral incisors and the anterior GL.

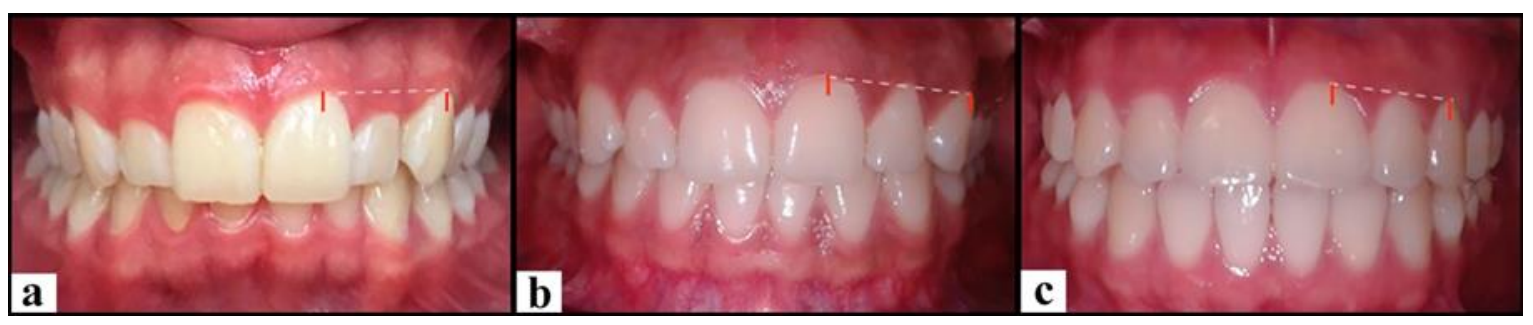

Figure 3 Gingival zenith level of the lateral incisors. (a): lateral incisor zenith coronal to the gingival line, (b): lateral incisor zenith apical to the gingival line, (c): lateral incisor zenith in coincidence with the gingival line.

The zeniths of the lateral incisors were principally located coronal to the GL (86.27\%), being that in 10 cases (10.78\%) this anatomical point was in coincidence with the GL and only $4(3.92 \%)$ had an apical location. The mean distance of lateral incisor zenith respect to the GL was of $0.82 \mathrm{~mm}(0.1-3.1 \mathrm{~mm} ; \pm 0.46 \mathrm{SD})$ (Table 4$)$.

Table 4 Distance between the gingival zenith of the lateral incisor and the gingival line.

\begin{tabular}{|l|l|l|l|}
\hline & $\begin{array}{l}\text { Minimum - Maximum } \\
\text { (mm) }\end{array}$ & $\begin{array}{l}\text { Mean } \\
\mathbf{( m m )}\end{array}$ & SD \\
\hline GZ of the 1.2 to right gingival line & $0.1-2$ & 0.79 & 0.39 \\
\hline GZ of the 2.2 to left gingival line & $0.2-3.1$ & 0.86 & 0.54 \\
\hline GZ of the LI to gingival line & $0.1-3.1$ & 0.82 & 0.46 \\
\hline
\end{tabular}

$\mathrm{GZ}=$ gingival zenith; $\mathrm{LI}=$ lateral incisor; $\mathrm{SD}=$ standard deviation.

As shown in Figure 4, there were not significant differences between male and female when compared the GZLs of their lateral incisors $(p=0.520)$. 


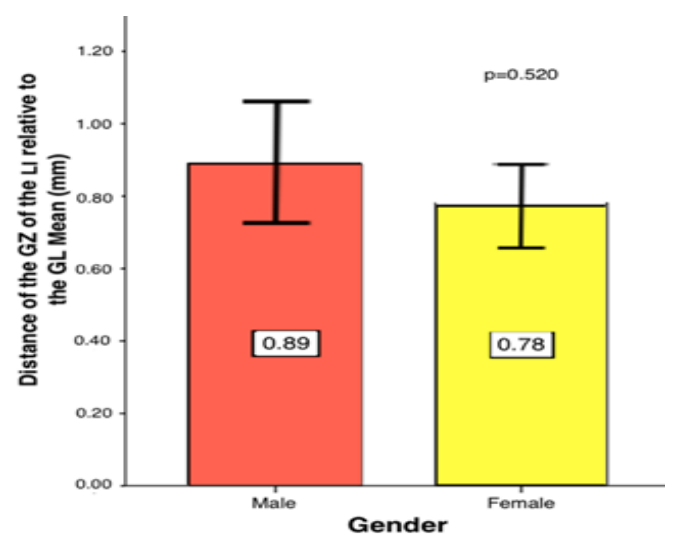

Figure 4 Gender based comparison of the distance between the gingival zenith of the lateral incisor and the gingival line.

\section{Discussion}

During an esthetic smile, the upper lip rises and exposes about 1 to $3 \mathrm{~mm}$ of the gingival tissues of the maxillary anterior teeth $[1,12]$. Contrastingly, the excessive exposure of the maxillary gingiva, beyond $3 \mathrm{~mm}$, can produce a antiesthetic smile called as "gummy smile", that can be corrected by orthodontic and/or surgical procedures [1,12,13]. Hence, in order to achieve optimal results in restorative, orthodontic or periodontal treatments in such area; it is important to take into consideration the gingival morphologic features, including the gingival zenith, line and level.

The present study verified that the distal location of the GZ is observed on the maxillary central and lateral incisors. These results confirms previous findings in which the GZ is distally displaced in most of these teeth $[2,3,5-7,9,14]$. On the other hand, there are contradictory information about the position of the GZ in canines. Although several dental literatures, including the present research, reveal that between $60 \%$ and $77.2 \%$ of the cuspids have a distal displacement of their GZ; other investigators observed that it coincides regularly with the long axis of these teeth and only Bharathi et al., verified its mesial displacement in $16.3 \%$ of their cases $[2,3,5-7,9,14,15]$.

Concerning quantification of the displacement of the GZ, in our study, the greater mean of displacement was verified in central incisors, followed by the lateral incisors and finally the minor values were noted in canines. These data corroborate previous observations, which indicate that the distal displacement of the GZ shows a decreasing pattern along the maxillary anterior teeth $[2,3,5-7,9,14,15]$. In addition, it has been previously reported that there is contralateral symmetry for the distal displacement of the gingival margin. Meanwhile, gender based comparisons have shown that the amount of displacement of the GZ can be either similar or different depending on the analyzed population[5,7,9,14,15]. For example, Flores-Jara et al. [7] and Nappe et al. [9] did not find differences of the amount of displacement among the genders, by contrast, the male individuals analyzed by Humagain et al. [15] demonstrated a higher magnitude of the distal position of the GZ in the lateral incisors compared to those teeth of the female participants. In the present study, we found that the displacement levels of the GZ of the central incisors were significantly higher in females.

Interestingly, studies have verified that between 42.7 to $51 \%$ of the premolars and even $29.3 \%$ of the first molars can be visualized during the smile [16,17], which implies that there are wide smiles that displays beyond the maxillary anterior teeth. In this context, to our knowledge only the study of Duran et al.[11] and the present report evaluated the characteristics of the GZ in the maxillary premolars. However, comparisons are impracticable because Duran et al. [11] established the positions of the premolar zenith according to the anterior gingival line. Meanwhile, our study assessed the location of the GZ relative to the long axis of these teeth and verified that like in the maxillary incisors and canines, this anatomical landmark is generally located in a distal position.

According to our analyses, the ascending gingival line was the most common in the maxillary anterior dentition, which is consistent with the finding of Zhang et al.[10] who evaluated the gingival line type in two-hundred young Chinese subjects. Additionally, we observed that in the premolar area the descending GL was the most prevalent, these results, which indicate that most of the second premolars had their GZ in a coronal position with respect to their counterpart of the first premolars, are similar to those previously described by Zhang et al.[10], who reported that the mean distance of GZ to distal extension of the anterior gingival line was significantly greater in second premolars than in first premolars. 
Regarding the characteristics of the GZL of the lateral incisors, Rufenacht[18] suggested that, ideally, for a class 1 occlusion, the gingival contours of the central incisors and canines should be located at the same level, being that on the lateral incisors it is slightly more coronal. Meanwhile, in class 2, division 2 malocclusions in which the laterals tend to overlap the distal aspects of the central incisors, the GZL of the lateral incisors is more apical compared with that of the central incisors and canines[18]. Despite the fact that we did not evaluate the occlusion class of the participants, our findings are in concordance with those of others investigators, who verified that the GZ of lateral incisors is more frequently located coronal to the GL, to approximately $1 \mathrm{~mm}$ and without significant differences among the genders $[2,3,5,8,10,15,19]$.

It is evident that some characteristics of the GZ are not universal and discrepancies may be result of differences among the studies including the samples size, the gender distribution, the evaluation method and the ethnicity of the participants. Concerning to the evaluation method, interestingly digital tools, like those employed for us, can help on the treatments planning, e.g., on the elaboration of surgical guides for procedures such as gingivectomies in which measures lower than $1 \mathrm{~mm}$ are practically impossible to establish by the human eye. Thereby, the data obtained in this study can be used clinically, during diagnosis and the esthetic treatment of maxillary teeth, especially for Andean patients. Further studies performed in these populations are warranted to analyze other features of the gingiva, including the gingival line angle and the interdental papillary heights in the anterior esthetic area.

\section{Conclusion}

The assessment of GZ, in an Andean populations from Ecuador, shows that:

- In the majority of the analyzed teeth, the GZs had a distal displacement with a decreasing pattern from the maxillary central incisor to canine.

- The ascending and descending GLs were the most frequent findings in the maxillary anterior teeth and premolars, respectively.

- In the majority of subjects, the GZs of the lateral incisors were located coronal to the anterior GL.

\section{Compliance with ethical standards}

\section{Disclosure of conflict of interest}

The authors declare that there is no conflict of interest.

\section{Statement of ethical approval}

The Research Committee of the School of Dentistry, University of Cuenca, Cuenca, Ecuador approved this study (UCDIF0-PROY-17-009).

\section{Statement of informed consent}

Informed consent was obtained from all individual participants included in the study.

\section{References}

[1] Kokich VG. Esthetics: The orthodontic-periodontic restorative connection. Semin Orthod. 1996; 2(1):21-30.

[2] Chu SJ, Tan JH, Stappert CF, Tarnow DP. Gingival zenith positions and levels of the maxillary anterior. J Esthet Restor Dent. 2009; 21(2): 113-20.

[3] Gowd S, ShankarT, Chatterjee S, Mohanty P, Sahoo N, et al. Gingival zenith positions and levels of maxillary anterior dentition in cases of bimaxillary protrusion: A morphometric analysis. J Contemp Dent Pract. 2017; 18(8): 700-704.

[4] Prichard J. Gingivoplasty, Gingivectomy, and Osseous Surgery. J Periodontol. 1961; 32(4): 275-282.

[5] Mattos CM, Santana RB. A quantitative evaluation of the spatial displacement of the gingival zenith in the maxillary anterior dentition. J Periodontol. 2008; 79(10): 1880-1885. 
[6] Bharathi SR, Cholan PK, Prakash P, Devapriya D, Devapriya A, Dhayanand JV, Sumi PS. Quantitative evaluation of the gingival zenith position and papilla proportions in the maxillary anterior dentition. Int J Pharma Bio Sci. 2017; 8(4): 495-501.

[7] Flores-Jara P, Delgado-Cotrina L, Webb-Linares J, Evangelista-Alva A. Estudio comparativo de dos instrumentos para determinar la ubicación del cenit gingival en dientes antero superiores. Rev. Estomatol Herediana. 2014; 23(3): 133-138.

[8] Pawar B, Mishra P, Banga P, Marawar P. Gingival zenith and its role in redefining esthetics: A clinical study. Journal of Indian Society of Periodontology. 2011; 15(2): 135-138.

[9] Nappe CE, Donoso FA, Díaz MI, Díaz J. Parámetros mucogingivales y dentarios en estudiantes de odontología chilenos. Rev Clín Periodoncia Implantol Rehabil Oral. 2015; 8(3): 228-233.

[10] Zhang Y, Le D, Hu WJ, Zhang H, Liang L, Chung KH, Cao Z. Assessment of dynamic smile and gingival contour in young Chinese people. International Dental Journal. 2015; 65(4): 182-187.

[11] Duran I, Ural C, Güler A, Yilmaz B, Tatar N. Determining the localization of premolar zenith positions according to the gingival line. Nigerian Journal of Clinical Practice. 2015; 18(2): 273-275.

[12] Ahmad I. Anterior dental aesthetics: Gingival perspective. British dental journal. 2005; 199(4): 195-202.

[13] Foley T F, Sandhu HS, Athanasopoulos C. Esthetic periodontal considerations in orthodontic treatment-The management of excessive gingival display. J Can Dent Assoc. 2003; 69(6): 368-372.

[14] Zagar M, Knezović Zlatarić D, Carek V. A quantitative evaluation of the distal displacement of the gingival zenith in the maxillary anterior teeth. Coll Antropol. 2010; 34: 225-228.

[15] Humagain M, Rokaya D, Srii R, Dixit S, Kafle D. Gender based comparison of gingival zenith esthetics. Kathmandu Univ Med J. 2016; 14(54): 148-152.

[16] Maulik C, Nanda R. Dynamic smile analysis in young adults. Am J Orthod Dentofacial Orthop. 2007; 132(3): 307315.

[17] Koenig R, Lavado A, Aguado J, Altamirano M, Gallardo G, Ramos E. Características de la sonrisa y nivel de satisfacción en estudiantes de la Facultad de Odontología de la Universidad de San Martín de Porres. Kiru. 2009; 6(2): 88-102.

[18] Rufenacht CR. Principles of esthetic integration. Chicago: Quintessence Pub. Co.; 2000. p. 154.

[19] Charruel S, Perez C, Foti B, Camps J, Monnet-Corti V. Gingival contour assessment: Clinical parameters useful for esthetic diagnosis and treatment. J Periodontol. 2008; 79(5): 795-801. 